\title{
PAX6 suppression of glioma angiogenesis and the expression of vascular endothelial growth factor $A$
}

\author{
Yi-Hong Zhou · Yuanjie Hu • Debra Mayes $\cdot$ Eric Siegel · \\ Jae G. Kim · Marlon S. Mathews · Nelson Hsu · Daniel Eskander • \\ Ong Yu $\cdot$ Bruce J. Tromberg $\cdot$ Mark E. Linskey
}

Received: 15 February 2009/ Accepted: 6 July 2009/Published online: 19 July 2009

(c) The Author(s) 2009. This article is published with open access at Springerlink.com

\begin{abstract}
We reported that PAX6 suppresses glioblastoma cell growth in vivo and anchorage-independent growth without significant alteration of cell proliferation in vitro, suggesting that PAX6 may alter the tumor microenvironment. Because we found that PAX6 downregulates expression of the gene encoding vascular endothelial growth factor A (VEGFA) in glioma cells, we used a subcutaneous xenograft model to verify PAX6 suppression of VEGFA-induced angiogenesis based on CD31-immunostaining of endothelial cells. The results showed a significant reduction of VEGFA at the transcription level in PAX6-transfected cells in xenografts and PAX6 has a suppressive effect on the microvascular amplification typically seen in glioblastoma. We showed that PAX6 suppression of VEGFA expression requires its DNA binding-
\end{abstract}

Y.-H. Zhou (ه) · Y. Hu · M. S. Mathews · N. Hsu ·

D. Eskander · O. Yu · M. E. Linskey

Department of Neurological Surgery, University of California,

Irvine, Irvine, CA 92697, USA

e-mail: yihongz@uci.edu

D. Mayes

Department of Pediatrics, Cincinnati Children's Hospital

Medical Center, Cincinnati, OH 45229, USA

E. Siegel

Department of Biostatistics, University of Arkansas for Medical

Sciences, Little Rock, AR 72205, USA

J. G. Kim · M. S. Mathews · B. J. Tromberg

Laser Microbeam and Medical Program, Beckman Laser

Institute, University of California, Irvine,

Irvine, CA 92697, USA

Y.-H. Zhou

UCI Medical Center, 101 The City Drive, Building 56, Suit 400,

Orange, CA 92868-3298, USA domain. The C-terminal truncation mutant of PAX6, however, did not show the dominant negative function in regulating VEGFA expression that it showed previously in regulating $M M P 2$ expression. In the glioma cell line U251HF, we further determined that blocking the PI3K/Akt signaling pathway with either adenoviral-mediated PTEN expression or LY294002 enhanced PAX6-mediated suppression of VEGFA in an additive manner; thus, PAX6mediated suppression of VEGFA is not via the canonical pathway through HIF1A. These two VEGFA-regulatory pathways can also be similarly modulated in another malignant glioma cell line, U87, but not in LN229 where the basal VEGFA level is low and PTEN is wild-type. PAX6 suppression of VEGFA appears to be blocked in LN229. In conclusion, our data showed that PAX6 can initiate in glioma cells a new signaling pathway independent of PI3K/ Akt-HIF1A signaling to suppress VEGFA expression.

Keywords Human GBM cell lines - Xenograft model · Angiogenesis · PAX6 · VEGFA · PTEN · PI3K signaling

$\begin{array}{ll}\text { Abbreviations } \\ \text { ELISA } & \text { Enzyme immunometric assay } \\ \text { AqRT-PCR } & \begin{array}{l}\text { Absolute quantitative reverse transcriptase- } \\ \text { polymerase chain reaction }\end{array} \\ \text { s.c. } & \begin{array}{l}\text { Subcutaneous } \\ \text { MI }\end{array} \\ \text { Modulated imaging }\end{array}$

\section{Introduction}

PAX6 is an early central-nervous-system (CNS) regulatory gene which has pleotropic effects during development of both the CNS and eye [1-3], and it contributes to both 
embryonic and adult neurogenesis as a multifunctional player regulating proliferation and differentiation through the control of expression of different downstream molecules in a highly context-dependent manner [4]. We have identified PAX6 as a glioma tumor suppressor gene, based on data showing PAX6 underexpression in higher grade gliomas as opposed to lower grade, in high tumorigenic glioma cell lines in comparison with low- and nontumorigenic ones, suppression of glioma-cell anchorageindependent growth, invasion, and tumorigenicity, and sensitization of glioma cells to detachment-induced stress [5-8]. Since glioma cells with stable expression of ectopic PAX6 cDNA showed dramatically suppressed growth in vivo, but no significant alteration of cell proliferation in vitro, it appears that PAX6 suppression of glioma tumorigenicity is mediated through changes in the tumor microenvironment, e.g. suppression of tumor angiogenesis.

Microvascular amplification is a histopathology hallmark in diagnosis of glioblastoma multiforme (GBM) - the highest grade and the most common type of glioma [9]. Vascular endothelial growth factor A (VEGFA) is the major angiogenic factor that is overexpressed in GBM [5]. An antiangiogenic agent effective in glioma, Bevacizumab (monoclonal antibody), blocks secreted VEGFA and significantly prolongs survival in patients with malignant glioma. Several small-molecule tyrosine-kinase inhibitors of the VEGFRA receptor show promise as well. Unfortunately despite promising overall results, more then $30 \%$ of patients initially fail to respond to anti-angiogenic treatment, and ultimately all patients will relapse and shortly thereafter die of their disease [10]. Understanding pathways abnormally suppressed/activated in glioma cells to affect VEGFA gene expression and VEGFA-induced angiogenesis will improve our understanding of resistance to anti-angiogenic therapies for malignant gliomas.

Enhanced expression of the VEGFA gene by glioma cells was found to occur through the stabilization of hypoxiainducible factor 1 alpha (HIF1A) under hypoxic conditions [11, 12], through the expression of the gene encoding tissue factor $(T F)$ [13-16], or through direct suppression of promoter activity mediated by the von Hippel-Lindau tumor suppressor gene (VHL) [17]. The action of phosphatase and tensin homolog (PTEN), a tumor suppressor, in suppressing VEGFA expression has been shown to occur via downregulation of HIF1A in a normoxic environment, which is mediated by blocking of the PI3K/Akt signaling pathwayan oncogene pathway active in malignant glioma known to be inactivated by PTEN [18].

By examining gene expressions in four GBMs and their adjacent normal tissues, we found that PAX6 levels were low while VEGFA levels were high in all four of the GBMs relative to adjacent normal tissues from the same patients [19], suggesting that PAX6 may suppress VEGFA gene expression in glioma cells. Here we report our study of PAX6 in glioma cell expression of VEGFA and glioma angiogenesis, and interestingly, co-regulation by PAX6 and PTEN of VEGFA expression in glioma cells.

\section{Materials and methods}

Cell lines, stable transfectants, and adenoviral vectors

The following cell lines were described in our previous study [7]: GBM cell lines U251HF, U87 and LN229, and stable PAX6 transfectants of U251HF established through transfection of a plasmid vector (pRC-CMV) expressing 1: wildtype PAX6, 2: C-terminal truncated PAX6 (PAX6-344), 3: mutant form of PAX6 with two mutations (R26G and I87R) in the DNA-binding domain (PD-dmt). The adenoviral vectors for PAX6 (Ad-PAX6) and GFP (Ad-GFP) were described in our previous study [6]. The adenoviral vector for PTEN (Ad-PTEN) and GFP (Ad-GFP) are from Dr. T. J. Liu (M.D. Anderson Cancer Center), which is the same adenovirus type-5 vector as that of Ad-PAX6. Cells were cultured in a DMEM/F12 medium supplemented with or without 5\% calf serum in $37^{\circ} \mathrm{C}$ under normoxic conditions with $5 \% \mathrm{CO}_{2}$. A hypoxic condition in experiment was established using a sealed chamber filled with $95 \% \mathrm{~N}_{2}$ and $5 \% \mathrm{CO}_{2}$.

\section{Subcutaneous (s.c.) implantation}

Cells of either U251HF or stable transfected U251HF expressing ectopic PAX6 (PAX6_2.2), were suspended in DMEM/F12 medium, and $5 \times 10^{6}$ cell in $50 \mu \mathrm{l}$ were subcutaneously (s.c.) injected into 4 to 6-week-old female NCrNu-M mice (Taconic, Hudson, NY). Three mice per group were treated, and s.c. injections were anterior to their right and left thighs. Mice were terminated at 25 days after the implantation, and the tumors were dissected, weighted and subjected to RNA extraction as described previously [20]. The s.c. experiment was repeated three times.

Immunohistochemistry analyses of s.c. xenografts

Mice with s.c. xenografts were euthanized, and xenografts were removed and embedded in cryopreservation medium on dry ice. The cryosections were cut $10 \mu \mathrm{m}$ thick and mounted on cleaned glass slides. After $1 \mathrm{~h}$ of $37^{\circ} \mathrm{C}$ drying, the sections were stored at $-80^{\circ} \mathrm{C}$. Before immunohistochemistry, sections were removed from $-80^{\circ} \mathrm{C}$, incubated 5-10 min in the oven at $37^{\circ} \mathrm{C}$, and fixed in $4 \%$ PFA for 20 $30 \mathrm{~min}$ at room temperature. Endothelial cells were stained immunohistochemically with primary rat anti-mouse PECAM-1 [CD31] antibody (1:300) from Millipore Corp (Temecula, CA), using hematoxylin to counterstain the 
nucleus, and detected with biotin-goat anti-mouse SS LINK and DAB detection system from BioGenex (San Ramon, $\mathrm{CA}$ ). Images were taken on the edge and central part of tumor sections, and blood vessel density (BVD) was calculated. We also performed double-staining of xenograft sections with rat anti-CD31 antibody (1:100) and rabbit anti-PAX6 antibody (1:1,000, kindly provided by Dr. Gary Philips), doublestaining with mouse anti-VEGF (1:100) and rabbit anti-PAX6 antibodies, and immunofluorescence detection with fluorescein anti-mouse $\operatorname{IgG}(\mathrm{H}+\mathrm{L})$ and rhodamine anti-rabbit $\operatorname{IgG}(\mathrm{H}+\mathrm{L})$ (Minipore Corp), using DAPI to counterstain the nucleus. Image analysis procedure and definitions of microvascular parameters were followed in reference [21].

\section{Modulated imaging (MI)}

The MI system has been developed at the Beckman laser institute and medical clinic, and the details of the system have been described previously [22]. We applied the MI technique to determine angiogenesis in vivo non-invasively. Briefly, the MI instrument uses patterned illumination and camera-based detection to obtain quantitative subsurface images of biological tissues over a wide field of view, with information on the optical properties of the tissue being imaged. Imaging at multiple wavelengths (between 650 and $980 \mathrm{~nm}$ ) provides quantitative measures of the in vivo concentrations of oxy- $(\mathrm{OHb})$, deoxy- (RHb) and total hemoglobin (THb) from subcutaneous xenografts. Oxygen saturation $\left(\mathrm{S}_{\mathrm{t}} \mathrm{O}_{2}\right)$ is a ratio of $\mathrm{OHb}$ to $\mathrm{THb}$.

Real-time absolute quantitative reverse transcription (AqRT-) PCR

The cDNA was reverse transcribed from 0.2 to $2 \mu \mathrm{g}$ of total RNA from the glioma cell lines, transfectants, and s.c. xenografts using 5 units of Superscript II reverse transcriptase (Invitrogen, Carlsbad, CA). The cDNA was diluted 30 times with $10 \mathrm{mM}$ Tris- $\mathrm{HCl}(\mathrm{pH}=7.5)$ and quantified for $V E G F A, V H L$, and internal reference genes $(G A P D H$ and ENO1) by real-time PCR with human-gene-specific primers (Ziren Research, Irvine, CA). Standard curves were used to determine quantities of the transcripts in cDNA samples. Absolute quantification of VEGFA and $V H L$ expressions relative to reference genes (GAPDH or ENO1) was achieved by using the single standard for both target and reference genes provided by Ziren Research LLC (Irvine, CA). The primer set for VEGFA was designed to amplify all seven transcription variants of $V E G F A$ reported in GeneBank. The primer set for GAPDH and ENO1 were designed to avoid amplification of pseudogenes that have high sequence similarities to the genes' cDNA sequences. The primer set for $V H L$ specifically amplifies VHL transcription variant 1 (NM_000551). Primer sequence information is available upon request to Ziren Research LLC (www.zirenresearch.com).

Western hybridization and VEGFA enzyme immunometric assay

Whole cell lysate extraction, western blotting, and PAX6 antibody were described previously [17]. Antibodies of Akt, Phopho-Akt (Ser473), and Phospho-GSK3b (Ser9) were from Cell Signaling Technology (Beverly, MA), while PTEN (A2B1) was from Santa Cruzis (Santa Cruz, California), and Actin was from EMD Bioscience (San Diego, CA). A human VEGFA enzyme immunometric assay (ELISA) kit (Assay Designs, Ann Arbor, MI) was used for quantification of the secretory VEGF165 in the conditioned media according to the manufacturer's instructions.

VEGFA promoter luciferase assay

The VEGFA promoter-luciferase constructs were a generous gift from V. P. Sukhatme (Beth Israel Deaconess Medical Center, Harvard Medical School) [17]. Transient transfection assay were performed using cells $(250,000$ cells per $35 \mathrm{~mm}$ dish plated 1 day before transfection) of $\mathrm{U} 251 \mathrm{HF}$ and three clones of U251HF expressing ectopic PAX6 with $0.1 \mu \mathrm{g}$ pRL-CMV expressing Renilla luciferase as an internal control together with $1 \mu \mathrm{g}$ VEGFA promoter constructs. Cell were cultured in serum-free medium for $48 \mathrm{~h}$ before harvesting cells for detection of luciferase activity using a Dual-Luciferase Reporter Assay System (Promega, Madison, WI) and a TD-20/20 Luminometer (Turner Designs, Sunnyvale, CA).

\section{Infection of Ad-PAX6 and Ad-PTEN in GBM cells}

Cells (U251HF, U87 and LN229) in triplicate were plated in 35-mm wells at a density of $4 \times 10^{5}$ cells per well and cultured overnight in DMEM/F12 medium supplemented with $5 \%$ calf serum. Cells were then infected in serum-free medium with adenovirus, 50 viral particles per cell for single infection and 25 viral particles per cell for double infection of Ad-PAX6 and Ad-PTEN together or 1 day apart. Cells were grown in normoxic or hypoxic $\left(95 \% \mathrm{~N}_{2}\right.$, $5 \% \mathrm{CO}_{2}$ ) for 24 or $48 \mathrm{~h}$ after infection, and then subjected to RNA extraction and cDNA synthesis for real-time AqRT-PCR. The medium was taken for VEGFA ELISA.

Statistical analysis

Data from real-time AqRT-PCR, ELISA, and luciferase assays were log-transformed to stabilize variance and reduce right-skewing. In some experiments, data were further 
normalized to the mean of the control treatment within each independent experiment replication. Depending on the design and number of groups, statistical analysis consisted of two-sample $t$-test, one-way ANOVA or randomized-block ANOVA. Multiple-comparison adjustments in ANOVA post-hoc analysis included Dunnett's procedure for pairwise comparisons of treatment groups to the control group, and Bonferroni's procedure for other pre-planned comparisons of interest. All analyses were conducted using SAS version 9.1.3 (The SAS Institute, Cary, NC).

\section{Results}

PAX6 suppress angiogenesis in glioma subcutaneous xenografts

We have tested multiple clones of the stable PAX6-transfected glioblastoma cell line U251HF in our previous study, and concluded that it was PAX6, not the clonal artifact, that suppressed U251HF cells' anchorage-independent growth, tumorigenicity and invasiveness, and that the vector-transfected clones showed no significant difference in these functional aspects to parental cells $[6,7]$. Here we investigated PAX6 regulation of glioma-cell-induced angiogenesis in subcutaneous (s.c.) xenografts derived from one of the transfectants (PAX6_2.2), in comparison with that from the parental cells. Consistent with our previous finding, the PAX6_2.2 cells form significantly smaller tumors compared to the parent cell U251HF after s.c. implantation in nude mice (Fig. 1a). Immunohistochemical (IHC) analysis of endothelial cell marker CD31, as shown in Fig. 1b, reveals that there is a significant reduction in the number and size of blood vessels in xenografts of PAX6_2.2 compared to that of $\mathrm{U} 251 \mathrm{HF}$. IHC analysis revealed severe central necrosis in $\mathrm{U} 251 \mathrm{HF}$ s.c. xenografts 25 days after implantation, and significantly fewer blood vessels in the central necrotic area compared to the periphery. We thus
A

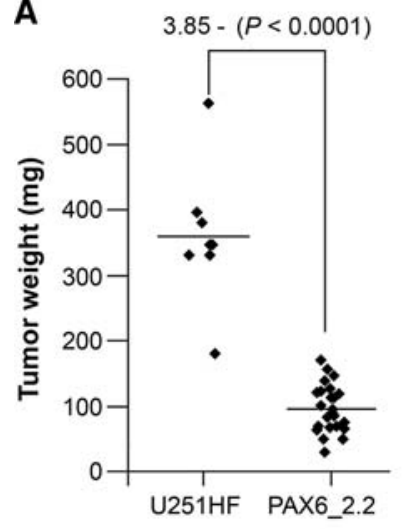

C

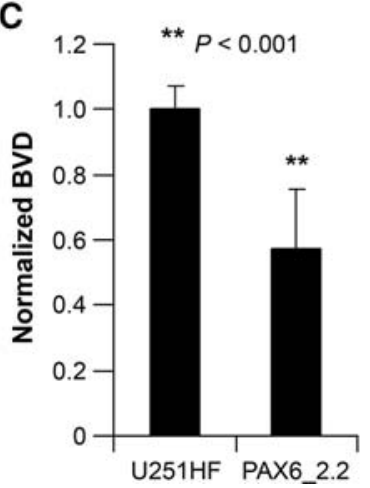

Fig. 1 PAX6 overexpression in glioma cell line U251HF suppresses cell tumorigenicity, tumor angiogenesis and VEGFA expression. a Comparison of the weights of U251HF $(n=8)$ and its PAX6 stable transfectant PAX6-2.2 xenografts $(n=24) 24$ days after subcutaneously implantation in nude mice. Fold change is $3.85 ; P<0.0001$ by two-sample $t$-test. b Immunofluroscent analysis of the xenografts from a for expression of PAX6 and CD31, with counterstaining of nuclei by DAPI. See [21] for image analysis procedure and definitions of microvascular parameters. c Comparison of blood vessel density
PAX6
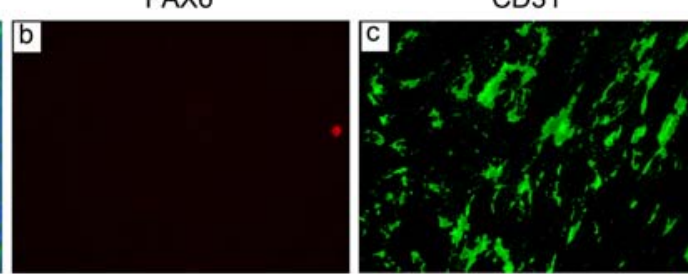

U251HF vessels

Number 48

Area $\quad 8143$

Perimeter 3392

Diameter 17.3
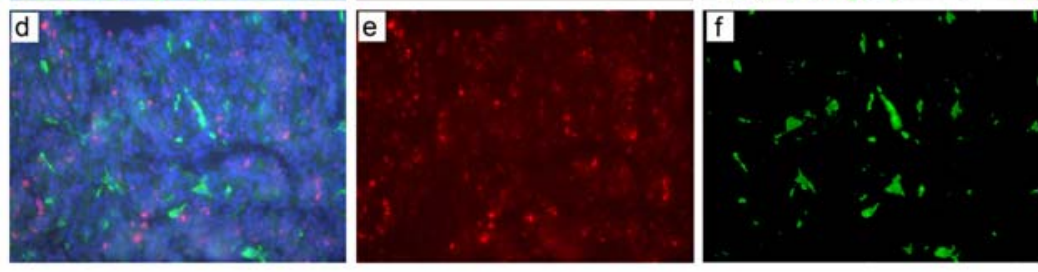

PAX6_2.2 vessels

Number 27

Area 2158

Perimeter 1045

Diameter 7.1
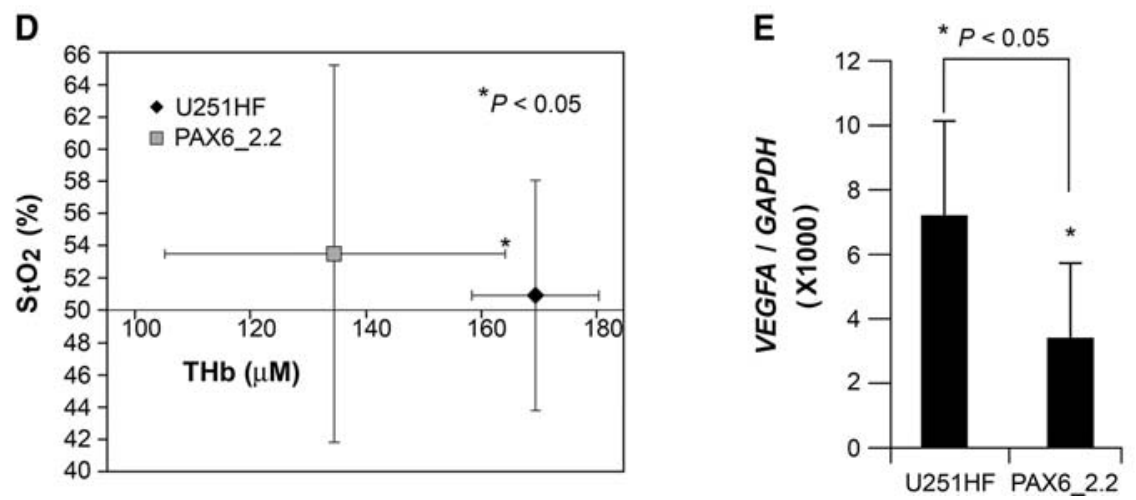

(BVD) normalized to peripheral region of U251HF tumor. d Comparison of total hemoglobin $(\mathrm{THb})$ concentration and tissue oxygen saturation $\left(\mathrm{StO}_{2}\right)$ in the two groups of s.c. xenografts (four tumors each group, two mice per group) by modulated imaging, all were subjected to CD31-IHC for BVD data above. Two-sample $t$-test $P$ values were 0.043 for $\mathrm{THb}$, and 0.70 for $\mathrm{StO}_{2}$, after Tukey-adjusted for comparisons among groups. e VEGFA expression in the s.c. xenografts from a by real-time AqRT-PCR. Fold change is 2.52 as shown; $P=0.025$ by two-sample $t$-test 
compared the blood vessel density (BVD) in tumor periphery regions of $\mathrm{U} 251 \mathrm{HF}$ xenograft to that from an overall region of xenografts from PAX6-2.2 that showed a more even distribution of blood vessels. As shown in Fig. 1c, there is a significantly lower BVD in tumor from PAX6_2.2 cells (Fig. 1c).

To confirm histological data, we analyzed a group of mice carrying the tumor with modulated imaging (MI) to monitor the blood uptake and consumption in tumor in vivo [22]. MI data revealed that PAX6-2.2 xenografts have significantly lower levels of both oxy-hemoglobin $(\mathrm{OHb})$ and deoxy-hemoglobin (RHb) compared to U251HF xenograft. Consequently, as shown in Fig. 1d, PAX6-2.2 xenografts have a significantly lower level of total hemoglobin $(\mathrm{THb}$ ) (sum of $\mathrm{OHb}$ and $\mathrm{RHb}$ ), but no significant difference in oxygen saturation $\left(\mathrm{S}_{\mathrm{t}} \mathrm{O}_{2}\right)$ (ratio of $\mathrm{OHb}$ to $\mathrm{THb}$ ). Therefore, increasing PAX6 level in U251HF cells does not change cellular metabolic activity, but does suppress tumor angiogenesis.

PAX6 suppresses VEGFA expression in vivo

We compared the VEGFA gene expression in the glioma cells in s.c. xenografts shown in Fig. 1, based on data from real-time AqRT-PCR using human-specific gene primers unable to amplify the murine homologues. As shown in Fig. 1e, we found a significantly lower level of VEGFA expression in the s.c. xenografts of PAX6_2.2 compared to those of U251HF; thus, PAX6-suppression of VEGFA takes place in vivo as well as in vitro.

We then quantified the VEGFA expression in $\mathrm{U} 251 \mathrm{HF}$ and PAX6_2.2 s.c. xenografts in a different experiment in which we remove the tumor at different times after implantation. As respectively shown in Fig. $2 \mathrm{a}$ and b, normalized VEGFA expression was found always higher, and PAX6 always lower, in U251HF xenografts compared to PAX6-2.2 xenografts that were grown for the same period of time in vivo. However, PAX6 in the PAX6-2.2 xenografts showed a $20 \%$ decrease in 15-day tumors compared to 11-day tumors (Fig. 2b), suggesting a negative selection pressure on the cells overexpressing PAX6 in vivo. Consistent with this finding, immunostaining of PAX6 in the 24-day PAX6_2.2 tumors showed loss of PAX6 signal in some tumor cells (Fig. 2d). Co-staining for PAX6 and VEGFA in PAX6_2.2 tumors showed distinct areas of cells with expression of either PAX6 or VEGFA but not both. This is consistent with the trends in Fig. 2a and $b$, which show that the decrease in PAX6 correlates

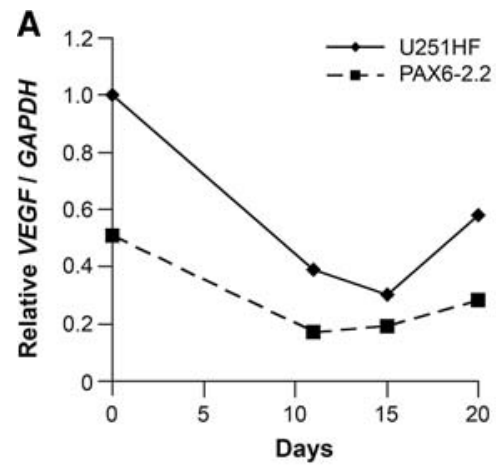

D

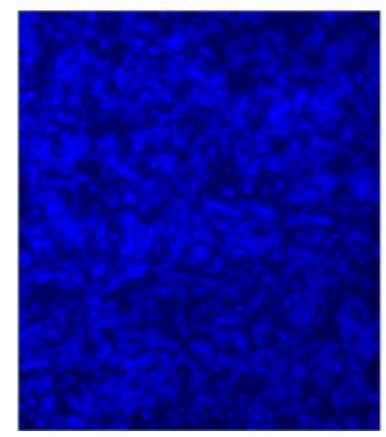

B

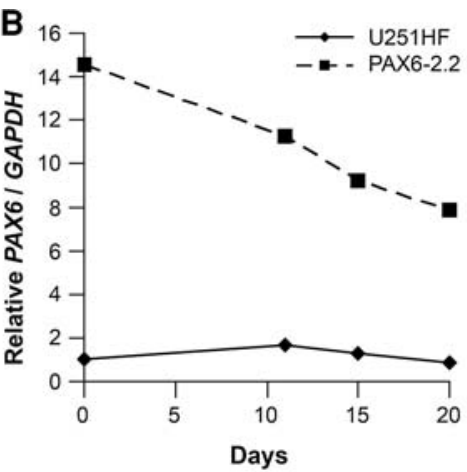

PAX6

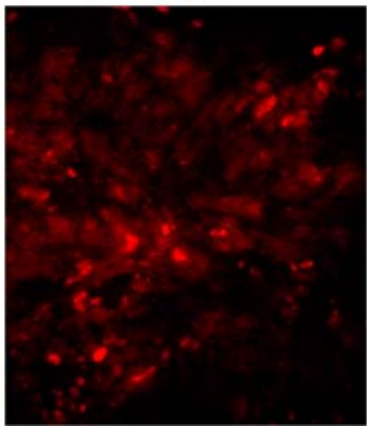

Fig. 2 Decrease of PAX6 is related to increase of VEGFA expression during tumor growth. a-b Comparison of PAX6 and VEGFA mRNA levels in U251HF and PAX6-2.2 cells grown in vitro or in vivo under nude mice skin at various time. The gene expression was quantified by real-time qRT-PCR, normalized to GAPDH, and compared with U251HF arbitrarily set to unity. c Comparison of tumor volumes that
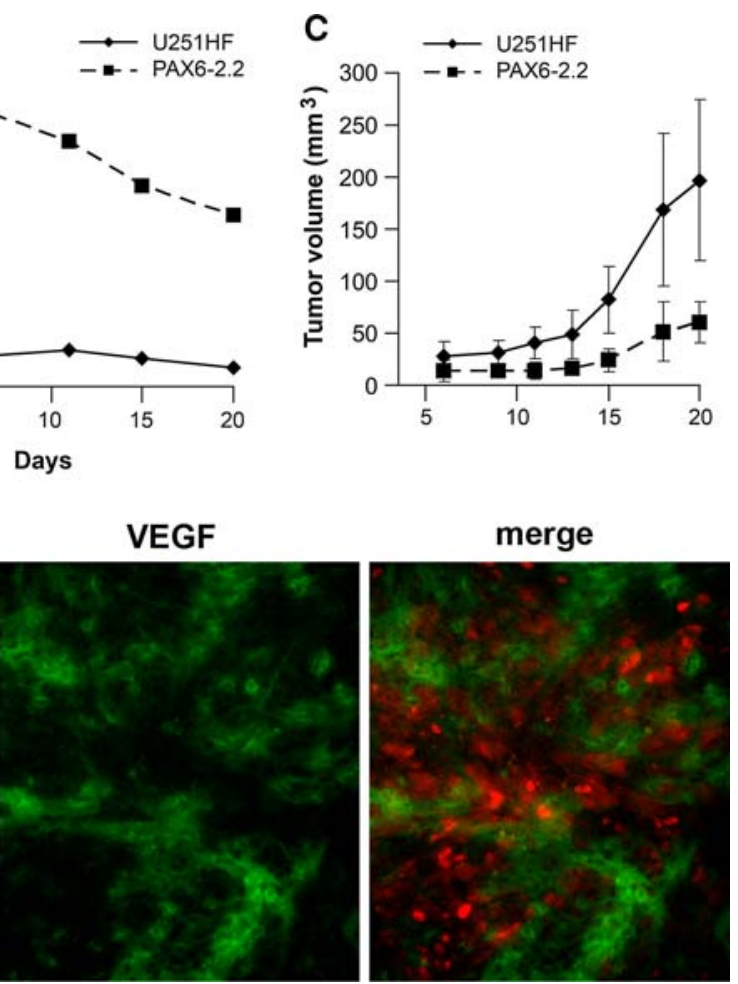

were measured 6 days after s.c. implantation of U251HF and PAX62.2. d Co-immunostaining PAX6_2.2 xenografts 24 days after implantation showing single pictures of PAX6 staining and VEGFA staining and merge picture of PAX6 and VEGFA, with cell nucleus counterstained by DAPI 
with the increase in VEGFA in overall tumor mass. Importantly, the transient changes in PAX6 and VEGFA mRNA levels in the tumors are related to increase of the tumor volumes (Fig. 2c). Overall, these results indicate that PAX6-mediated suppression of VEGFA expression is responsible for the suppression of tumorigenicity of gliomas, which is shown to be related with suppression of angiogenesis.

PAX6 downregulation of VEGFA expression at transcription level requiring its DNA-binding domain

We then examined in vitro cell cultures under normoxic condition for changes on secretion of VEGFA in conditioned medium by $\mathrm{U} 251 \mathrm{HF}$ and multiple clones of its stable transfectants of wild-type or mutant forms of PAX6. As shown in Fig. 3a, it is the expression of wild-type PAX6, not the mutant forms of PAX6, that changed the VEGFA level secreted by the modified cells.

We further investigated PAX6 regulation of VEGFA promoter activity. The transcriptional regulation of the $V E G F A$ promoter seems to play the pre-eminent role in the control of VEGFA expression, and several response elements within the $V E G F A$ promoter region have been characterized in vitro. A key region of the VEGFA promoter, located at approximately -930 from the transcription initiation site, is a target for a number of transcription factors, including HIF1A, TP53, VHL, and SP1. We thus investigated VEGFA promoter activity by transfection of luciferase constructs containing various regions of the VEGFA promoter $(2.6,1.5,0.35$ and $0.2 \mathrm{~kb}$ as described previously [17]) in U251HF and its PAX6-transfectants. Since the suppressive effects of PAX6 on the VEGFA promoter were observed in all promoter constructs in PAX6_2.2 cells (data not shown), we focused on examining the $0.2 \mathrm{~kb}$ VEGFA promoter, which contains the -52 / +144 promoter sequence. This region does not contain any of the binding sites for HIF1A, TP53, VHL, and SP1. As shown in Fig. $3 b$, the $-52 /+144$ VEGFA promoter activity was lower in all three PAX6-transfectants compared to the parental cell line $\mathrm{U} 251 \mathrm{HF}$; the average of activities relative to $\mathrm{U} 251 \mathrm{HF}$ among the three transfectants was $40 \%$ $(P<0.0001)$. Overall, the data showed that PAX6 suppressed VEGFA expression in glioma cells under normoxic in vitro and subcutaneous in vivo conditions.

PAX6-mediated regulation of VEGFA expression is independent of HIF1A or VHL

Although VEGFA expression is regulated via complex pathways, regulation of HIF1A synthesis and stability has been considered to be the central mechanism underlying regulation of VEGFA expression. The tumor suppressor PTEN was reported to suppress VEGFA expression by
A

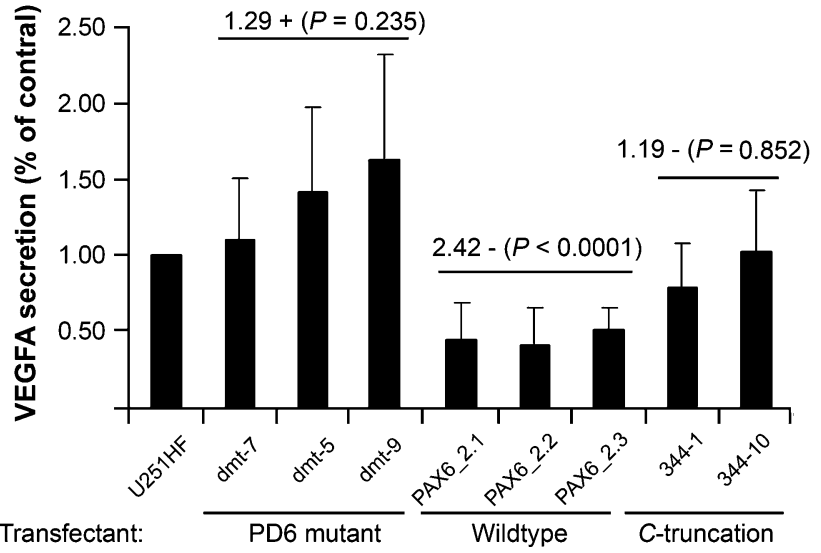

B VEGFA $0.2 \mathrm{~kb}$ promer construct
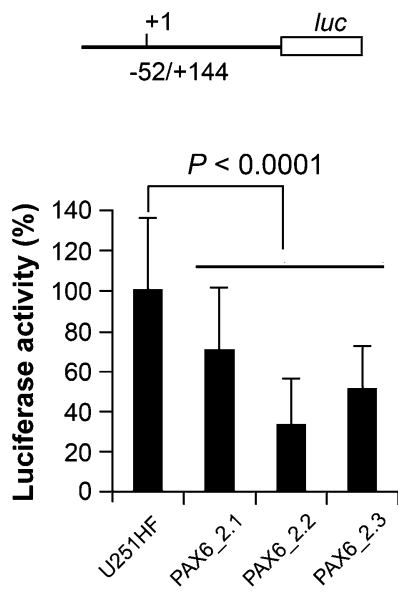

Fig. 3 PAX6 regulation of VEGFA expression. a Comparison of secreted VEGFA quantified by VEGF-165 ELISA from condition medium of cells expressing transfected cDNA constructs of wild-type or two mutant forms of PAX6 (dmt and 344) to the parental glioma cell U251HF, which was arbitrarily set to unity. Bonferroni adjusted $P$-value with fold of increase $(+)$ and decrease $(-)$ were shown with averages for the clones use for comparison. On top depicts the PAX6 mutants in functional domains, with PAI and RED and HD represent three DNA-binding domain of PAX6, with two point mutations in the paired domain R26G and I87R reported to cause loose of in vitro DNA binding ability [7]. b Luciferase assays of $V E G F$ promoter activities in U251HF and three of U251HF PAX6 transfected clones, with comparison of $-52 /+144 V E G F$ promoter activities between parental and PAX6-transfected cells. Mean and SD were from 4 to 8 repeats of transfection and luciferase assay, which were carried out in 2-4 independent experiments 
blocking the PI3K/Akt signaling pathway with subsequent reduction of HIF1A protein level under a normoxic environment [18]. We thus examined the possible influence of PAX6 on regulation of VEGFA through the PI3K/Akt signaling pathway. Western blots were used to detect the activation of the PI3K/Akt signaling pathway in response to adenoviral-mediated overexpression of PAX6 in glioma cells. The result showed that PAX6 did not attenuate the phosphorylation of Akt and GSK3B (Fig. 4a), nor the level of HIF1A (Fig. 4b), in contrast to what was found with adenoviral-mediated overexpression of PTEN in glioma cells.

Data from real-time AqRT-PCR quantification of the $V H L$ gene expression revealed a high expression of $V H L$ in U251HF cells: more than 4-fold higher than the level of the reference gene ENOI (Fig. 4c, first column). We further examined $V H L$ expression $48 \mathrm{~h}$ following infection of U251HF cells with adenovirus of PAX6 (Ad-PAX6), with Ad-PTEN, or with both, in two independent experiments. Compared to the mock infection, Ad-PAX6 transfection produced a reduction in the $V H L / E N O 1$ expression ratio of $25 \%$ when used alone (Fig. 4c, second column; $P=0.035$ ) and $27 \%$ when co-transfecting with Ad-PTEN (Fig. 4c, fourth column; $P=0.026$ ), whereas transfection with AdPTEN alone produced a $36 \%$ increase in $V H L / E N O 1$ (Fig. 4c, third column; $P=0.027$ ). Since the VHLexpression changes in $\mathrm{U} 251 \mathrm{HF}$ cells did not support a suppressive function on VEGFA expression following overexpression of PAX6 alone or together with PTEN, it implies that VHL is unlikely to contribute to PAX6 suppression of VEGFA expression.
PAX6 suppression of VEGFA expression was enhanced by blocking PI3K/Akt signaling pathway

Based on data shown above, PAX6 does not activate the PI3K/Akt signaling pathway, which suggests that the mechanism by which PAX6 suppresses VEGFA differs from that by which PTEN suppresses VEGFA. In agreement with this conclusion, we observed that co-transfection of U251HF cells with Ad-PAX6 and Ad-PTEN enhanced suppression of VEGFA at the levels of both mRNA (Fig. 5a) and secreted VEGFA (Fig. 5b), while no change was seen from transfection with Ad-GFP, excluding possible viral effect. Statistical analysis showed that PAX6 and PTEN work on mRNA levels in an additive manner, i.e. with no significant synergism or antagonism (Table 1). The significant main effects coupled with the lack of significant interaction shows that co-transfection with PAX6 and PTEN produces significantly more suppression of VEGFA expression than either mono-transfection.

Consistent with PTEN blockage of PI3K/Akt signaling to mediate suppression of VEGFA expression, treatment of cells with the PI3K inhibitor, LY294002, also gave rise to reduction of VEGFA expression in a dose-dependent manner. Co-treatment of Ad-PAX6 and LY294002 further increased the suppression of VEGFA. As shown in Fig. 5a, treatment of U251HF cells with LY294002 alone for $48 \mathrm{~h}$ decreased VEGFA expression, to $72 \%$ (normalized to $G A P D H$ ) and $64 \%$ (normalized to ENOI) with a $2 \mu \mathrm{M}$ dose, and to $65 \%$ (normalized to $G A P D H$ ) and $49 \%$ (normalized to $E N O 1$ ) with a $10 \mu \mathrm{M}$ dose. In the same culture condition, infection of Ad-PAX6 alone at a lower dose (10
A

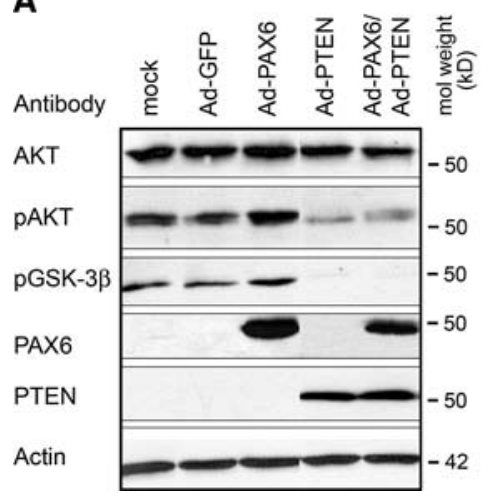

B

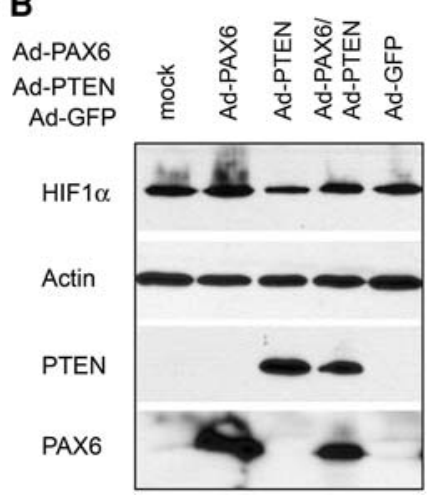

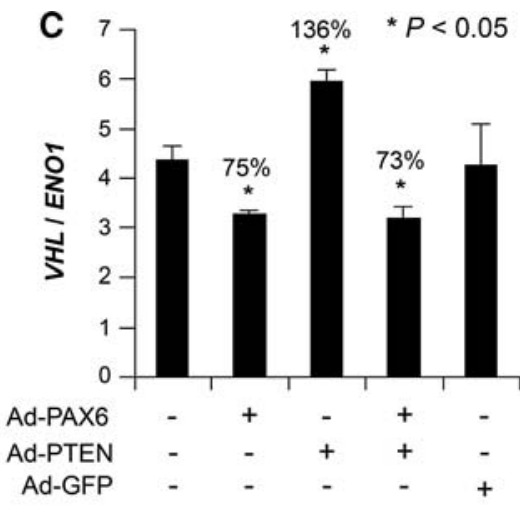

Fig. 4 PAX6 does not alter PI3K/Akt signaling and HIF1A level in glioma cells under normoxic condition. By Western blot assay, phosphorylation status of Akt and the downstream target GSK-3 $\beta$ (a) and HIF1A level (b) were shown unchanged in U251HF cells $48 \mathrm{~h}$ after treatment of cells with of Ad-PAX6. Analysis of cells with AdPTEN infection was positive control for antibodies, and detections of Actin as control equal protein loading while PAX6 and PTEN for positive infections. c AqRT-PCR revealed the level of VHL expression relative to ENO1 in U251HF cells with or without adenoviral infections. The mean and SD are based on data of two independent experiments, with viral dose of 10 viral partial per cell, cultured for $48 \mathrm{~h}$ under serum-free condition after $1 \mathrm{~h}$ of infection. $P$-values are shown for comparisons to mock infection, and are computed using Dunnett's ANOVA post-hoc procedure 

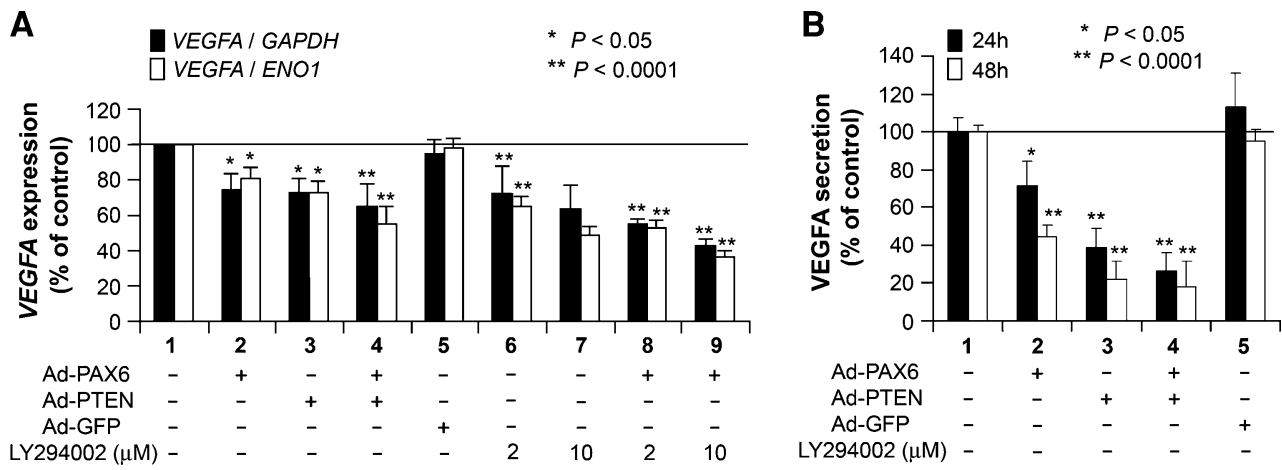

Fig. 5 PAX6 suppression of VEGFA expression was enhanced by coexpression of PTEN or blocking Akt-signaling pathway in U251HF cells under normoxic condition. Comparison of VEGFA expression in

Table 1 Statistical analysis of VEGFA expressions normalized to either GAPDH or ENO1 in U251HF cells response to Ad-PAX6, AdPTEN, and LY294002 by post-hoc comparisons and contrasts of the treatments

\begin{tabular}{lccc}
\hline Effect on VEGFA expressions & $F$-statistics $^{\mathrm{a}}$ & $\mathrm{DF}^{\mathrm{b}}$ & $P$ values $^{\mathrm{c}}$ \\
\hline PAX6 main effect \pm PTEN & 30.03 & $(1,24)$ & $<0.0001$ \\
& 11.49 & & $<0.0001$ \\
PTEN main effect \pm PAX6 & 56.81 & $(1,24)$ & $<0.0001$ \\
& 13.93 & & $<0.0001$ \\
PAX6-by-PTEN interaction & 0.67 & $(1,24)$ & 0.42 \\
& 1.99 & & 0.17 \\
PAX6 main effect \pm LY294002 & 34.12 & $(1,24)$ & $<0.0001$ \\
& 34.09 & & $<0.0001$ \\
LY294002 main effect \pm PAX6 & 136.10 & $(2,24)$ & $<0.0001$ \\
& 36.60 & & $<0.0001$ \\
PAX6-by-LY294002 interaction & 0.34 & $(2,24)$ & 0.72 \\
& 0.42 & & 0.66 \\
\hline
\end{tabular}

${ }^{a}$ Contrast $F$-statistics for indicated effect. Top: VEGFA/ENO1; bottom: VEGFA/GAPDH

${ }^{\mathrm{b}}$ Contrast degrees of freedom (DF) for indicated effect, expressed as (effect DF, error DF)

${ }^{c}$ Contrast $P$ values for indicated effect. Top: VEGFA/ENO1; bottom: VEGFA/GAPDH

viral particles per cell) decreased the expression of VEGFA down to $74 \%$ (normalized to GAPDH) and $81 \%$ (normalized to ENO1). However, combined treatment with AdPAX6 and LY294002 enhanced the overall suppression of VEGFA, yielding expression levels of 55\% (normalized to $G A P D H$ ) or $53 \%$ (normalized to ENOI) with $2 \mu \mathrm{M}$ LY294002, and expression levels of 44\% (normalized to GAPDH) or $37 \%$ (normalized to ENO1) with $10 \mu \mathrm{M}$ LY294002. Statistical analysis showed that PAX6 and LY294002 work in an additive manner, i.e. with no significant synergism or antagonism (Table 1).

Similar effects on the enhanced suppression of VEGFA by co-overexpression of PAX6 and PTEN were observed
U251HF cells after treatment Ad-PAX6 and/or Ad-PTEN, and/or PI3K inhibitor LY294002 at mRNA level (a) and at secreted protein level (b). Bonferroni adjusted $P$ value were shown

in another glioma cell line, U87. Interestingly, such enhancement can be achieved only when the cells were co-infected by Ad-PAX6 and Ad-PTEN, regardless of whether conditions were normoxic or hypoxic (Fig. 6). Changes in cell viability after vial infection were determined to be minimal based on a Trypan Blue exclusion assay.

Infection of another glioma cell line LN229 with AdPAX6 alone or together with Ad-PTEN at a lower dose (10 viral particles per cell) decreased the expression of VEGFA down to $70 \%$ (normalized to GAPDH or ENOI) (Fig. 6b). But since Ad-GFP also caused a similar level of reduction when normalized to $G A P D H$, it brings the attention to viral effect in LN229. Trypan Blue exclusion assay showed that LN229 is sensitive to adenoviral infection as well as LY294002 treatment, which apparently cause reductions of ENO1 expression and increase of VEGFA/ENOI values in Ad-GFP and LY294002 treatments. In contrast to AdPAX6, Ad-PTEN slightly increased VEGFA expression in LN229 by about 1.5 fold (Fig. 6b). Glioma cell line LN229 is one of the few glioma cell lines that has a wild-type form of the PTEN gene. We compared the basal VEGFA expression in LN229 with other glioma cell lines with mutant PTEN. As shown in Fig. 6c, basal VEGFA expression in LN229 is low; 4 or 9\% of U251HF normalized to GAPDH or ENO1, respectively, and 2 or $6 \%$ of U87 normalized to GAPDH or ENO1, respectively. Thus VEGFA expression in LN229 apparently is under partial suppression, and can be further suppressed by PAX6 such that expression is nearly blocked, but cannot be further suppressed by increasing PTEN-blockage of PI3K/Akt signaling.

\section{Discussion}

Our data show, for the first time, that PAX6 acts as a tumor suppressor in glioma cells by suppressing angiogenesis, and that VEGFA is a target of PAX6-mediated pathways in 
A

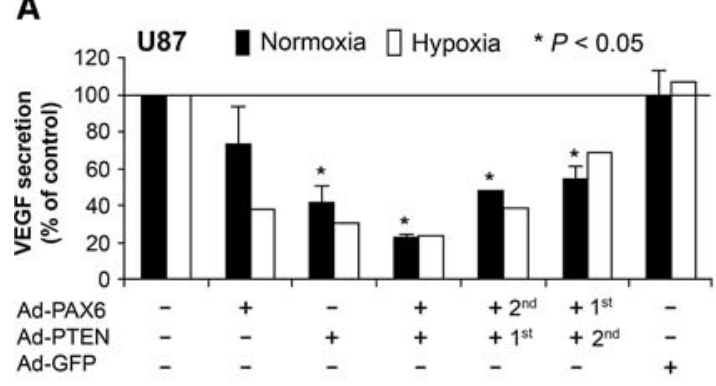

C

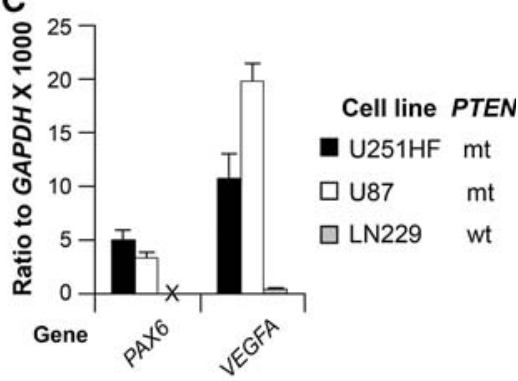

B

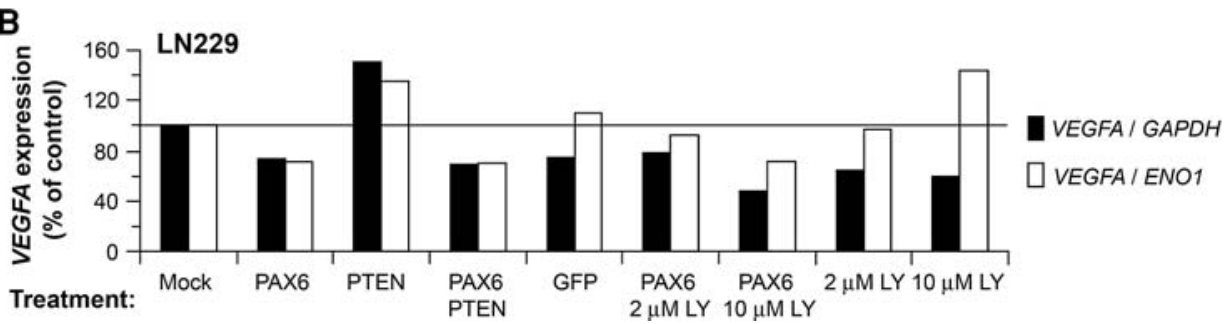

Fig. 6 Regulation of VEGFA expression in U87 and LN229 by PAX6 or blocking of PI3K/Akt signaling. a VEGF-165 ELISA data on the secreted VEGFA protein in condition medium of cells infected with Ad-PAX6 alone or together with Ad-PTEN simultaneously or separate with 1 day apart, under normoxic and hypoxic conditions. Mean and SD were from 1 to 2 independent experiments, with $P$-values shown for comparison to mock infection. b Comparison of

a normoxic condition. We found that PAX6 suppression of $V E G F A$ is under a mechanism different from the canonical mechanism involving HIF1A and VHL. Consequently, an additive suppressive effect on VEGFA expression was seen by introduction of both PAX6 and PTEN or PAX6 and PI3K inhibitor. Yet our data from this study did not identify a mediator of the PAX6 reduction of VEGFA promoter activity and consequent mRNA expression. Through transfection of different forms of PAX6 mutants, we determined that PAX6 needs its DNA-binding domain to suppress VEGFA expression, but this study did not solve the question of whether PAX6 functions directly to bind the VEGFA promoter or indirectly via another transcription factor. Data from this study reveal that PAX6 mediated a novel mechanism for regulating VEGFA expression that is independent of the PI3K/Akt-HIF1A-VHL pathway.

In this study, we used the s.c. xenograft glioma model to study the direct effect of PAX6 on angiogenesis. In this model, we can avoid complications delineating the mechanisms affecting angiogenesis in the brain, where angiogenesis can also be affected by microglia/microphage infiltration $[22,24]$. Since PAX6 was found to suppress expression of the gene encoding chemokine ( $\mathrm{C}-\mathrm{C}$ motif) ligand 2 (CCL2) in glioma cell lines [25], using a s.c. xenograft model allowed us to exclude the microglia effect on angiogenesis in an intracranial xenograft model. With regards to the potential regulation of inflammation-induced angiogenesis by PAX6 expression in glioma cells, the
VEGFA expression in LN229 cells after treatment Ad-PAX6 and/or Ad-PTEN, and/or PI3K inhibitor LY294002 at mRNA level. c comparison of PAX6 and VEGFA expressions in U251HF, U87 and LN229. X marks zero expression value for PAX6 in LN229; PTEN status ( $m t$, mutant or $w t$, wild type) in these cell lines is based on information in [23]

intracranial xenograft model will be used. Identification of PAX6 regulation of VEGFA expression and the underlying mechanisms will provides new clues to dissect the complexity of mechanisms underlying glioma angiogenesis.

Acknowledgments This work was supported in part by the Arkansas Cancer Research Center at the University of Arkansas for medical sciences, and University of California, Irvine, Committee on Research Award.

Open Access This article is distributed under the terms of the Creative Commons Attribution Noncommercial License which permits any noncommercial use, distribution, and reproduction in any medium, provided the original author(s) and source are credited.

\section{References}

1. Simpson TI, Price DJ (2002) Pax6; a pleiotropic player in development. Bioessays 24:1041-1051

2. Mansouri A, Hallonet M, Gruss P (1996) Pax genes and their roles in cell differentiation and development. Curr Opin Cell Biol 8:851-857

3. Sander M, Neubuser A, Kalamaras J, Ee HC, Martin GR, German MS (1997) Genetic analysis reveals that PAX6 is required for normal transcription of pancreatic hormone genes and islet development. Genes Dev 11:1662-1673

4. Osumi N, Shinohara H, Numayama-Tsuruta K, Maekawa M (2008) Concise review: Pax6 transcription factor contributes to both embryonic and adult neurogenesis as a multifunctional regulator. Stem Cells 26:1663-1672 
5. Zhou YH, Tan F, Hess KR, Yung WK (2003) The expression of PAX6, PTEN, vascular endothelial growth factor, and epidermal growth factor receptor in gliomas: relationship to tumor grade and survival. Clin Cancer Res 9:3369-3375

6. Zhou YH, Wu X, Tan F, Shi YX, Glass T, Liu TJ, Wathen K, Hess KR, Gumin J, Lang F, Yung WK (2005) PAX6 suppresses growth of human glioblastoma cells. J Neurooncol 71:223-229

7. Mayes DA, Hu Y, Teng Y, Siegel E, Wu X, Panda K, Tan F, Yung WK, Zhou YH (2006) PAX6 suppresses the invasiveness of glioblastoma cells and the expression of the matrix metalloproteinase-2 gene. Cancer Res 66:9809-9817

8. Chang JY, Hu Y, Siegel E, Stanley L, Zhou YH (2007) PAX6 increases glioma cell susceptibility to detachment and oxidative stress. J Neurooncol 84:9-19

9. CBTRUS, Central Brain Tumor Registry of the United States (2005) Statistical report: primary brain tumors in the United States, 1997-2002. CBTRUS, Chicago

10. Norden AD, Drappatz J, Wen PY (2008) Antiangiogenic therapy in malignant gliomas. Curr Opin Oncol 20:652-661

11. Wiesener MS, Munchenhagen PM, Berger I, Morgan NV, Roigas J, Schwiertz A, Jurgensen JS, Gruber G, Maxwell PH, Loning SA, Frei U, Maher ER, Grone HJ, Eckardt KU (2001) Constitutive activation of hypoxia-inducible genes related to overexpression of hypoxia-inducible factor-1alpha in clear cell renal carcinomas. Cancer Res 61:5215-5222

12. Damert A, Machein M, Breier G, Fujita MQ, Hanahan D, Risau W, Plate KH (1997) Up-regulation of vascular endothelial growth factor expression in a rat glioma is conferred by two distinct hypoxia-driven mechanisms. Cancer Res 57:3860-3864

13. Guan M, Jin J, Su B, Liu WW, Lu Y (2002) Tissue factor expression and angiogenesis in human glioma. Clin Biochem 35:321-325

14. Hamada K, Kuratsu J, Saitoh Y, Takeshima H, Nishi T, Ushio Y (1996) Expression of tissue factor correlates with grade of malignancy in human glioma. Cancer 77:1877-1883

15. Poon RT, Lau CP, Ho JW, Yu WC, Fan ST, Wong J (2003) Tissue factor expression correlates with tumor angiogenesis and invasiveness in human hepatocellular carcinoma. Clin Cancer Res 9:5339-5345
16. Takano S, Tsuboi K, Tomono Y, Mitsui Y, Nose T (2000) Tissue factor, osteopontin, alphavbeta3 integrin expression in microvasculature of gliomas associated with vascular endothelial growth factor expression. Br J Cancer 82:1967-1973

17. Mukhopadhyay D, Knebelmann B, Cohen HT, Ananth S, Sukhatme VP (1997) The von Hippel-Lindau tumor suppressor gene product interacts with $\mathrm{Sp} 1$ to repress vascular endothelial growth factor promoter activity. Mol Cell Biol 17:5629-5639

18. Gomez-Manzano C, Fueyo J, Jiang H, Glass TL, Lee HY, Hu M, Liu JL, Jasti SL, Liu TJ, Conrad CA, Yung WK (2003) Mechanisms underlying PTEN regulation of vascular endothelial growth factor and angiogenesis. Ann Neurol 53:109-117

19. Zhou YH, Hess RK, Liu L, Linskey ME, Yung WKA (2005) Modeling prognosis for patients with malignant astrocytic gliomas: quantifying the expression of multiple genetic markers and clinical variables. Neuro Oncol 7:485-494

20. Wesseling P, van der Laak JA, de Leeuw H, Ruiter DJ, Burger PC (1994) Quantitative immunohistological analysis of the microvasculature in untreated human glioblastoma multiforme. Computer-assisted image analysis of whole-tumor sections. J Neurosurg 81:902-909

21. Cuccia DJ, Bevilacqua F, Durkin AJ, Tromberg BJ (2005) Modulated imaging: quantitative analysis and tomography of turbid media in the spatial-frequency domain. Opt Lett 30:13541356

22. Platten M, Kretz A, Naumann U, Aulwurm S, Egashira K, Isenmann S, Weller M (2003) Monocyte chemoattractant protein-1 increases microglial infiltration and aggressiveness of gliomas. Ann Neurol 54:388-392

23. Ishii N, Maier D, Merlo A, Tada M, Sawamura Y, Diserens AC, Van Meir EG (1999) Frequent co-alterations of TP53, p16/ CDKN2A, p14ARF, PTEN tumor suppressor genes in human glioma cell lines. Brain Pathol 9:469-479

24. Goede V, Brogelli L, Ziche M, Augustin HG (1999) Induction of inflammatory angiogenesis by monocyte chemoattractant protein1. Int J Cancer 82:765-770

25. Zhou YH, Glass T, Yung WKA (2001) PAX6 down regulate the expression of PDGFRA and MCP-1 genes in glioblastoma cells. Neuro Oncol 3:312 